\title{
Prognostic Implications of Novel $\beta$ Cardiac Myosin Heavy Chain Gene Mutations That Cause Familial Hypertrophic Cardiomyopathy
}

\author{
Ryuichiro Anan, ** Gottfried Greve, "Ludwig Thierfelder," Hugh Watkins," \\ Carlo Vecchio, ${ }^{\star *}$ Hirohisa Shono, ${ }^{\ddagger}$ Shoichiro Nakao, ${ }^{*}$ Hiromitsu Tanaka, ${ }^{\ddagger}$ Adolph Mares, Jr.," \\ Jeffrey A. Towbin, ${ }^{3}$ Paolo Spirito, * * Robert Roberts, J. G. Seidman, * and Christine E. Seidman" \\ * Department of Genetics and Howard Hughes Medical Institute, Harvard Medical School, Boston, Massachusetts 02115; \\ ${ }^{\ddagger}$ First Department of Internal Medicine, Kagoshima University, Kagoshima 890, Japan; ${ }^{\S}$ Department of Medicine, \\ Baylor College of Medicine, Houston, Texas 77030; "Department of Medicine, Brigham and Women's Hospital and Harvard \\ Medical School, Boston, Massachusetts 02115; 'Department of Cardiological Science, St. George's Hospital Medical School, \\ London SW17 ORE, UK; and **Division of Cardiology, Ospedali Galliera, Genoa, Italy
}

\begin{abstract}
Three novel $\beta$ cardiac myosin heavy chain (MHC) gene missense mutations, Phe513Cys, Gly716Arg, and Arg719Trp, which cause familial hypertrophic cardiomyopathy (FHC) are described. One mutation in exon 15 (Phe513Cys) does not alter the charge of the encoded amino acid, and affected family members have a near normal life expectancy. The Gly716Arg mutation (exon 19; charge change of +1 ) causes FHC in three family members, one of whom underwent transplantation for heart failure. The Arg719Trp mutation (exon 19; charge change of -1) was found in four unrelated FHC families with a high incidence of premature death and an average life expectancy in affected individuals of 38 yr. A comparable high frequency of disease-related deaths in four families with the Arg719Trp mutation suggests that this specific gene defect directly accounts for the observed malignant phenotype. Further, the significantly different life expectancies associated with the Arg719Trp vs. Phe513Cys mutation $(P<0.001)$ support the hypothesis that mutations which alter the charge of the encoded amino acid affect survival more significantly than those that produce a conservative amino acid change. ( J. Clin. Invest. 1994. 93:280-285.) Key words: cardiac hypertrophy $\bullet$ missense $\bullet$ survival $\bullet$ sudden death
\end{abstract}

\section{Introduction}

Molecular genetic studies have begun to decipher the broad clinical spectrum of familial hypertrophic cardiomyopathy (FHC). ${ }^{1}$ Chromosomes 1 (1), 11 (2), 14 (3), and 15 (4) have been demonstrated to contain disease loci that cause FHC, but specific mutations have only been identified in the $\beta$ cardiac myosin heavy chain (MHC) gene on chromosome 14. Allelic heterogeneity further contributes to the complexity of the ge-

Address correspondence to Dr. Christine E. Seidman, Harvard Medical School, Alpert Building, Room 533, 200 Longwood Avenue, Boston, MA 02115. 1993.

Received for publication 6 July 1993 and in revised form 27 August

1. Abbreviations used in this paper: FHC, familial hypertrophic cardiomyopathy; MHC, myosin heavy chain.

J. Clin. Invest.

(c) The American Society for Clinical Investigation, Inc.

0021-9738/94/01/0280/06 \$2.00

Volume 93, January 1994, 280-285 netic basis of FHC. Many different missense mutations have been identified in the head or head/rod junction of the $\beta$ cardiac MHC gene in families with this disorder.

Identification of the full complement of $\beta$ cardiac MHC gene mutations that cause FHC may help in both the elucidation of the pathophysiology of this disorder and the assessment of prognosis. Analyses of genotype and phenotype may in particular provide insights into the molecular basis for cardiac dysfunction in this condition. Studies have detected little correlation between the morphologic characteristics of the hypertrophic phenotype seen in four different $\beta$ cardiac MHC gene mutations (5), suggesting that the extent and distribution of the cardiac hypertrophy observed in affected individuals reflects the interactions of other genes and/or environmental factors. In contrast, there is recent evidence that genotype can account for the variable patient outcomes observed in different families with FHC. Different missense mutations within the $\beta$ cardiac MHC gene appear to be associated with different life expectancies in affected individuals (6). Two mutations have been identified ( Val606Met [6] and Leu908Val; [7]) that produce a conservative change of the encoded amino acid. The prognosis of individuals with these mutations appears better than that observed in individuals with a $\beta$ cardiac MHC gene mutation that encodes an amino acid with a different charge.

To further elucidate those features of $\beta$ cardiac MHC gene mutations that impact on the clinical manifestations of FHC, we have studied additional families. Three novel mutations have been identified in six unrelated families. Analyses of clinical data were informative for two mutations. We report a novel conservative mutation (Phe513Cys) that has little effect on patient survival and a nonconservative mutation (Arg719Trp) that is associated with a significant reduction in life expectancy.

\section{Methods}

Clinical evaluation. Consent was obtained in accordance with the local institutional review committees for human subject investigations. Clinical, electrocardiographic and echocardiographic assessments of six families were performed as described previously $(3,8)$. The diagnosis of hypertrophic cardiomyopathy was based on the demonstration of unexplained left, right, or biventricular hypertrophy. Blood was obtained from all family members for genetic analyses.

Clinical records and family history were obtained to determine the number of disease-related deaths, and the age at death or current age for all affected individuals in each family. For Kaplan-Meier survival curves, individuals who were resuscitated from cardiac arrest were scored as deceased at the age of their event. Kaplan-Meier product limit survival curves were produced as described (6).

Detection of mutations. Lymphocytes isolated from peripheral blood samples were used to establish a transformed cell line with Ep- 
stein-Barr virus as described previously (3). $\beta$ cardiac MHC gene or mRNA sequences were amplified from a proband's transformed lymphocytes by sequential rounds of PCR (9). Amplified sequences were hybridized to RNA probes transcribed from a normal gene and RNase A protection assays performed as described (6). Analyses were limited to sequences encoding amino acid residues $1-1,080$, which were examined using both sense and antisense riboprobes. Amplified sequences that yielded abnormal RNA cleavage patterns were reanalyzed with new DNA or mRNA isolates to exclude artifacts arising from PCR.

DNA obtained from probands in two families (120 and 134) were scanned for mutations in exon 19 and 20 by chemical cleavage as described elsewhere $(10,11)$. These exons were amplified together and heteroduplexes were formed by a denaturing and reannealing reaction between the PCR template and both sense and antisense radiolabelled probes transcribed from a normal gene. The heteroduplexes were modified separately with hydroxylamine and osmium tetroxide, and the modified products were cleaved with piperidine. Products were resolved by electrophoresis in an $8 \%$ polyacrylamide gel.

Mutations identified by RNase protection assays or chemical cleavage were subjected to dideoxy nucleotide sequence analysis. Where possible, the nucleotide change identified by sequencing was independently confirmed by demonstration of a predicted change in restriction enzyme site or by chemical cleavage of the exon containing a mutation. Amino acid sequences were deduced from DNA sequences. Nucleotide and amino acid residues are numbered according to Jaenicke (12). The following primers were used to amplify exons 15 and 19:

Forward (15F): ACTCACACCCACTTTCTGACTGCTC

Reverse (15R): GAATTCAGGTGGTAAGGCCAAAGAG

Forward (19F): CTCACAGACTCCTCCTACTTCCTTC

Reverse (19R): GCCTGGCTCCCCCTGTTCTATGAGC

\section{Results}

Clinical results. The pedigrees of three FHC families (AX, AZ, and $\mathrm{AI}$ ) are shown in Fig. 1. All affected members had typical clinical features of this disorder (Table I). Family AX is of Japanese ancestry. Of 11 affected individuals 3 have died; in 1 death was disease-related; in 2 death was noncardiac and occurred in the 8th decade. Family AZ resides in Italy. Three affected individuals have been identified and two (II-1 and III-1) have developed progressive left ventricular wall thinning with systolic dysfunction. Individual II-1 underwent cardiac transplantaton for intractable heart failure at age 42 . The biventricular weight of the explanted heart was $400 \mathrm{~g}$ and coronary anatomy was normal. Histologic examination demonstrated interstitial fibrosis (less than $1.5 \mathrm{~cm}$ in length) and myocyte hypertrophy. Family AI is a small family from the UK. Two affected individuals are alive, one of whom was resuscitated from sudden death at age 19.

Clinical studies of three other FHC families (101, 120, and $134)$ have been previously reported $(8,13,14)$. In addition to the typical signs and symptoms of FHC found in affected members of these families, a high incidence of sudden death and disease-related death has been observed. Of 28 affected individuals in family 101, 19 died prematurely (before age 58); death was sudden in 10 individuals and disease-related in 9 individuals. Of 7 affected individuals from family 120 , sudden death occurred in 2 and disease-related death occurred in 1. Death occurred in 12 of 23 affected members of family 134; in 10 , death was sudden; in 2, death was disease-related; one survivor in this family was resuscitated from sudden cardiac death at age 15 .

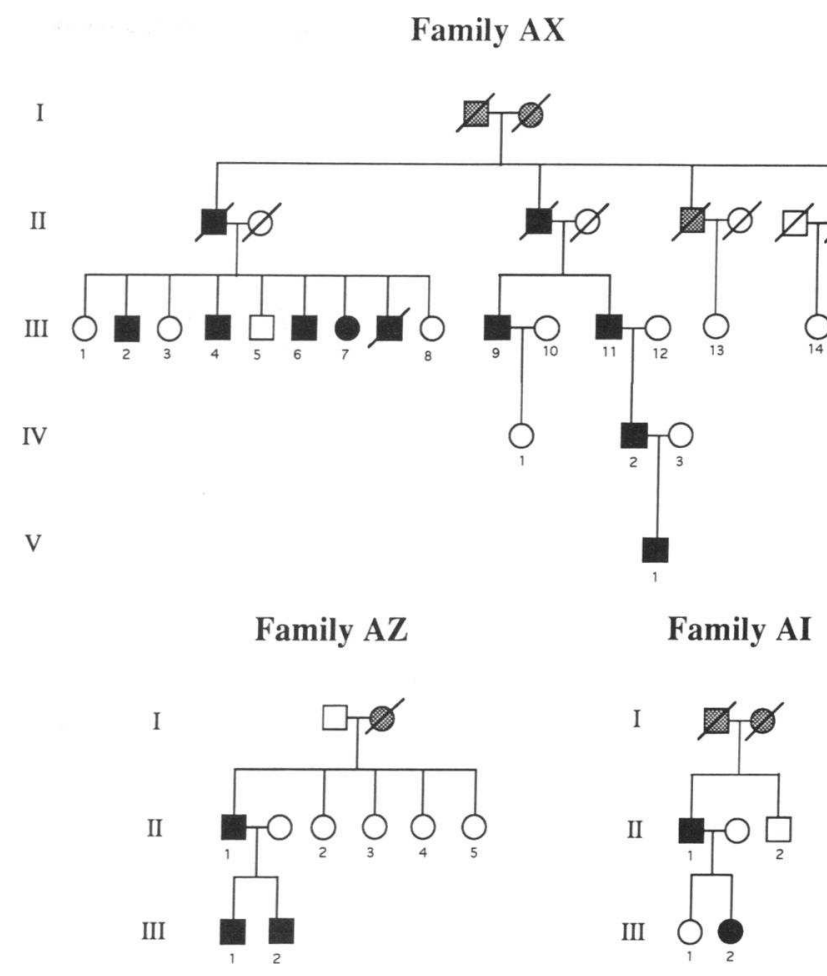

Figure 1. Pedigrees of three FHC families. Symbols denote sex and disease-status: box, male; circle, female; solid, affected; clear, unaffected; speckled, unknown; slashed, deceased. Individuals identified with a pedigree number were clinically and genetically studied.

Mutation identification. The $\beta$ cardiac MHC sequences of a proband from families AX, AZ, AI, and 101 were screened for missense mutations using $\mathrm{RNase}$ protection assays as described previously $(6,9)$. Because most $\beta$ cardiac MHC gene mutations have been identified in the amino terminal half of the gene (6), sequences encoding amino acid residues $1-1,080$ were analyzed. PCR-amplified sequences that produced unusual RNase protection cleavage products were subcloned and their nucleotide sequences determined. The same abnormal product was observed in RNase protetion assays of probands from families $\mathrm{AI}$ and 101 , suggesting that they shared the same $\beta$ cardiac MHC gene mutation. Nucleotide sequence analyses confirmed that in the probands from families AI and 120 the cytosine at position 2241 (exon 19) has been mutated to a thymine residue (Fig. $2 A$ and data not shown).

The C2241T transition abolishes an MspI site found in unaffected individuals (Fig. $3 A, \operatorname{Arg} 719 T r p$ ) and is also identified by chemical cleavage of PCR-amplified exon 19 and 20 DNA (data not shown). These methods permit independent confirmation of genotypes identified by RNase protection assays. Restriction enzyme digestion or chemical cleavage of PCR-amplified exon 19 sequences were used to study all affected individuals in families $\mathrm{AI}$ and 101 and in two additional FHC families (120 and 134). All members of families AI, 101, 120, and 134 with clinical evidence of FHC were demonstrated to have the $\mathrm{C} 2241 \mathrm{~T}$ mutation.

A second mutation in exon 19 was identified by RNase protection assay. A transition (guanine 2232 to adenine, Fig. 2 $B$ ) was identified in the proband of family AZ. This mutation creates a novel SfcI restriction enzyme site (Fig. $3 B$, Gly716Arg). PCR amplification of exon 19 of the $\beta$ cardiac 
Table I. Clinical Features of FHC in Affected Individuals from Three Families

\begin{tabular}{|c|c|c|c|c|c|c|c|c|c|c|c|}
\hline \multirow[b]{2}{*}{ Subject } & \multirow[b]{2}{*}{ Age/sex } & \multicolumn{3}{|c|}{ Clinical symptoms } & \multicolumn{3}{|c|}{ Electrocardiogram } & \multicolumn{4}{|c|}{ 2-D echocardiogram } \\
\hline & & $\begin{array}{l}\text { Chest } \\
\text { pain }\end{array}$ & Dyspnea & Syncope & BBB & ABN Q & $\begin{array}{c}\text { LVH } \\
+/- \text { ST }\end{array}$ & $\begin{array}{c}\text { MAX } \\
\text { LVWT }\end{array}$ & RVH & SAM & $\begin{array}{c}\text { LA } \\
\text { SIZE }\end{array}$ \\
\hline AX III-2 & $72 \mathrm{M}$ & - & III & - & - & - & - & 14 & - & - & 38 \\
\hline AX III-4 & $65 \mathrm{M}$ & - & III & - & - & - & + & 22 & - & + & 54 \\
\hline AX III-6 & $57 \mathrm{M}$ & - & I & - & - & - & + & 17 & - & + & 45 \\
\hline AX III-7 & $54 \mathrm{~F}$ & - & III & - & - & - & + & 17 & - & - & 32 \\
\hline AX III-9 & $75 \mathrm{M}$ & - & II & - & - & - & - & 19 & - & - & 40 \\
\hline AX III-11 & $72 \mathrm{M}$ & - & I & - & - & + & + & 22 & - & - & 33 \\
\hline AX IV-2 & $40 \mathrm{M}$ & - & I & - & - & + & - & 17 & - & - & 43 \\
\hline $\mathrm{AX} V-1^{*}$ & $8 \mathrm{M}$ & - & I & - & - & - & - & $9^{*}$ & - & - & 20 \\
\hline AZ II-1 & $43 \mathrm{M}$ & - & III & - & $\mathbf{L}$ & - & - & 25 & - & - & 60 \\
\hline AZ III-1 & $22 \mathrm{M}$ & - & II & + & $\mathbf{L}$ & - & - & 17 & - & - & 55 \\
\hline AZ III-2 & $16 \mathrm{M}$ & - & I & - & - & - & + & 20 & - & - & 44 \\
\hline AI II-1 & $58 \mathrm{M}$ & - & I & - & B & - & - & 21 & - & - & 50 \\
\hline AI III-2 & $22 \mathrm{~F}$ & - & I & + & B & - & - & 26 & - & - & 58 \\
\hline
\end{tabular}

The degree of dyspnea present was based on the New York Heart Association functional classification. BBB denotes bundle-branch block (R, right; L, left; B, bifasicular); ABN Q, abnormal Q wave; LVH +/- ST, left ventricular hypertrophy with voltage criteria and/or repolarization changes. Max LVWT, maximal thickness of the left ventricular wall $(\mathrm{mm})$; RVH, right ventricular hypertrophy; SAM, systolic anterior motion of the mitral valve, LA, left atrial size (mm). ${ }^{*}$ FHC was diagnosed in individual AX V-1 (age 8) based on an increased LVWT for his body surface area of $1.08 \mathrm{~m}^{2}$.

MHC gene from all members of family AZ demonstrated this SfcI site only in those individuals with FHC.

RNase protection assays of the proband from family AX identified a mutation in exon 15 that changes the thymine at position 1624 to a guanine (Fig. $2 \mathrm{C}$ ). Since this transversion does not effect a restriction enzyme site, all family members were studied with RNase protection assays. Only clinically affected individuals were found to have two novel fragments resulting from cleavage of the normal 171-bp product into products of sizes 130 and 41 bp (Fig. $3 C$ ).

The evidence that these nucleotide changes accounts for FHC is based on three observations. First, each nucleotide change alters an encoded amino acid. The C2241T mutation in families AI, 101, 120, and 134 encodes an Arg719Trp change; the G2232A mutation in family AZ encodes a Gly716Arg change; the T1624G mutation in family AX encodes a Phe513Cys change. Two of these missense mutations are nonconservative and alter the charge (Arg719Trp, -1 and Gly716Arg, +1) of the encoded amino acid. Second, each of these missense mutations alters an amino acid that has been conserved in all mammalian myosin molecules studied (data not shown). The Phe513Cys mutation alters a myosin heavy chain residue that has been stringently conserved in all vertebrates. Third, these mutations have not been found in analyses of more than 200 normal chromosomes. Fourth, there is complete concordance between clinical status and genotype in each family.

Survival analyses. To define the natural history of hypertrophic cardiomyopathy due to each of these missense mutations, we compared the survival of affected individuals from each family. Too few individuals with the Gly716Arg mutation were present in Family AZ to construct meaningful KaplanMeier survival curves. However we note that one of three affected individuals has required cardiac transplantation for progressive left ventricular wall thinning associated with intractable heart failure. Four unrelated families with FHC resulting from an Arg719Tr mutation were identified. Survival of affected individuals in each of these families was similar and
A

B

G A T C G A T C

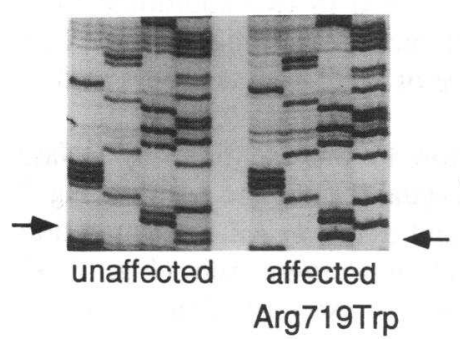

G A T C G A T C

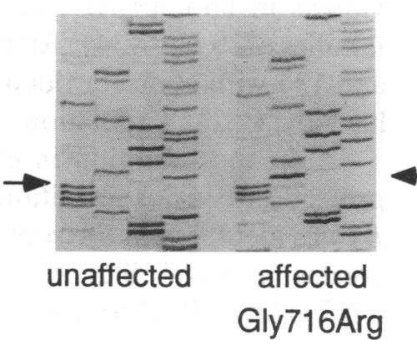

C

G A T C G A T C

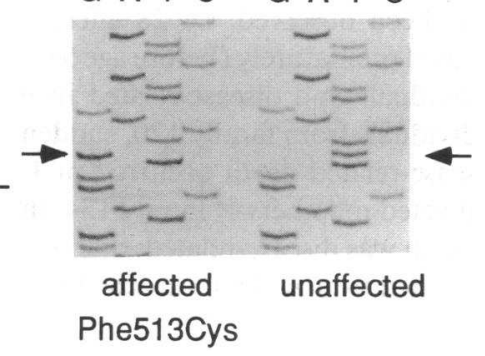

Figure 2. Nucleotide sequence of $3 \beta$ cardiac MHC gene mutations that cause FHC. The normal and mutated sequence (arrows) obtained from an affected and unaffected individual from one family are shown. (A) The C2241T mutation in families AI, 101, 120, and 134 encodes an Arg719Trp change. $(B)$ The G2232A mutation in family $A Z$ encodes a Gly716Arg change. (C) The T1624G mutation in family $\mathrm{AX}$ encodes a Phe513Cys change. 
A

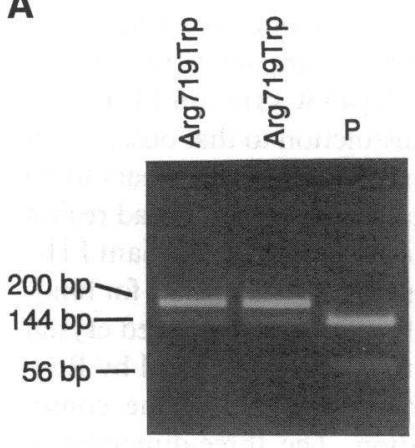

B

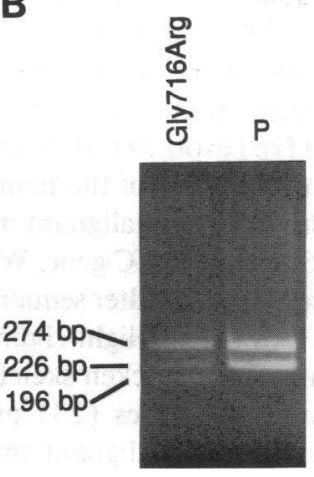

C
$P \quad P$ P $\stackrel{\stackrel{+}{=}}{\stackrel{x}{x}}$

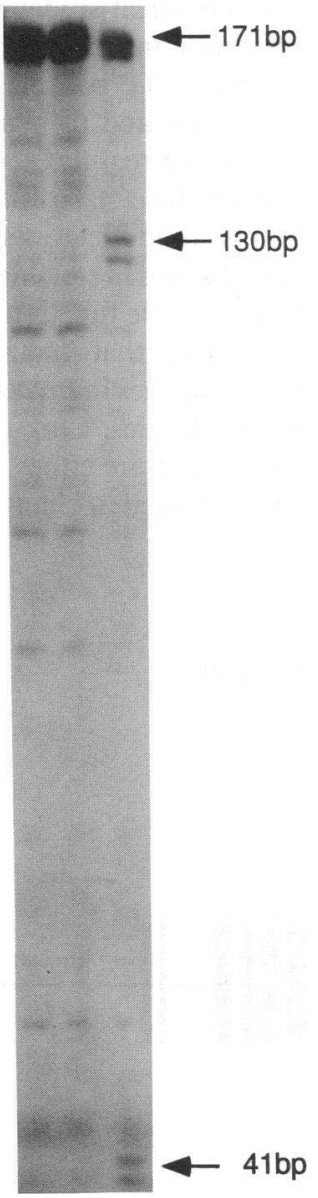

Figure 3. A representation of techniques used to genotype family members for $\beta$ cardiac MHC gene mutations. ( $A$ ) Arg719Trp (exon 19): A 200-bp fragment of the $\beta$ cardiac MHC gene was amplified using primers numbered B19F and B19R (Methods) and digested with MspI. Sequence from clinically unaffected individuals $(P)$ generates fragments of 144 and $56 \mathrm{bp}$; individuals with the Arg719Trp mutation lack the MspI site on one allele, resulting in the 200-bp as well as normal fragments. ( $B$ ) Gly7 16Arg (exon 19): A 500-bp fragment of the $\beta$ cardiac MHC cDNA was amplified as using primers $1925 \mathrm{~F}$ and $2424 \mathrm{R}(6)$ and digested with SfcI. Analysis of cDNA from an unaffected individual $(P)$ yields products of sizes 274 and $226 \mathrm{bp}$. DNA from individuals with the Gly716Arg mutation have an additional SfcI site that cleaves the 226-bp fragment into fragments sized

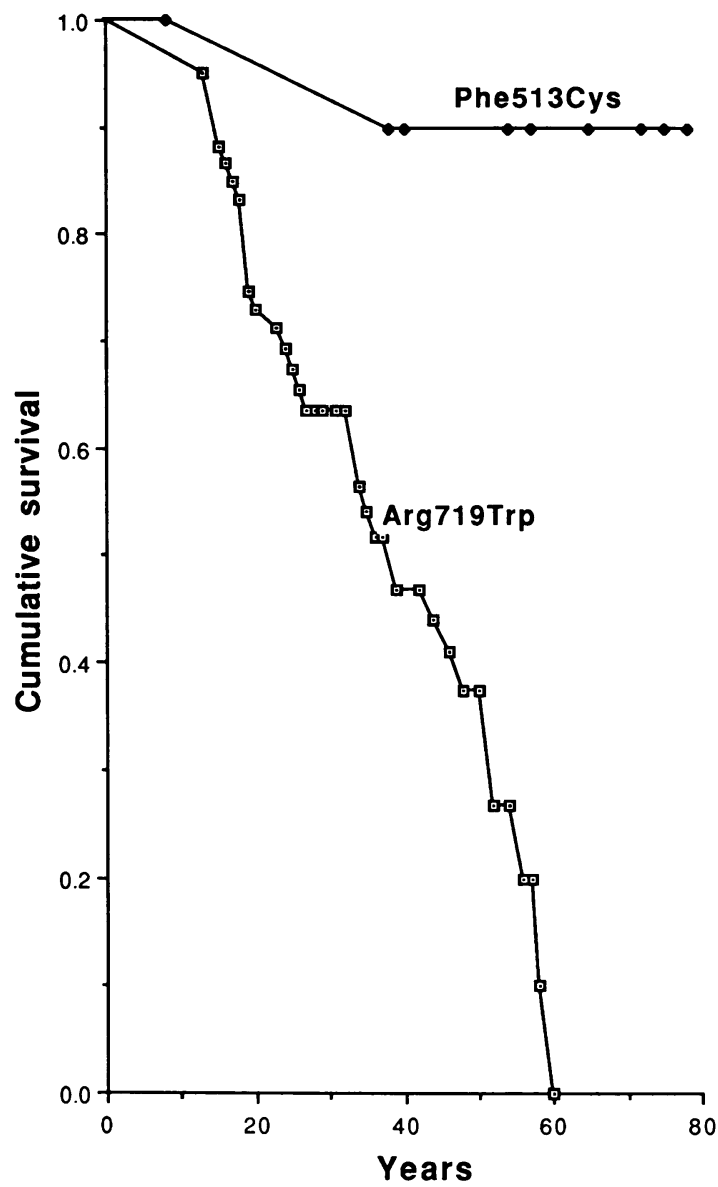

Figure 4. Kaplan-Meier product-limit curves for the survival of individuals bearing the Arg719Trp or Phe513Cys mutation. Data from four unrelated families with the Arg719Trp mutation are combined, as no differences were detected between these families. A significant difference $(P<0.001)$ in life expectancy was observed in individuals with the Arg719Trp vs. the Phe513Cys mutation.

averaged only $38 \mathrm{yr}$. In contrast, survival was near normal in the 11 affected individuals from family $\mathrm{AX}$ with the conservative Phe513Cys mutation. Fig. 4 demonstrates the significantly different survival $(P<.001)$ associated with FHC due to the Arg719Trp mutation vs. the Phe513Cys mutation. Life expectancy associated with the Phe513Cys is comparable to that seen in other conservative $\beta$ cardiac MHC gene mutations (6). The Arg719Trp mutation portends a survival that is comparable to that seen in FHC due to an Arg403GIn mutation; both mutations are associated with a particularly poor prognosis.

\section{Discussion}

We report three previously unidentified $\beta$ cardiac $\mathrm{MHC}$ gene mutations that cause FHC in six families. Two of these muta-

at 196 and $30 \mathrm{bp}$ (not visible). (C) Phe513Cys (exon 15) is detected by RNase protection assays. Amplified DNA is hybridized to a ${ }^{32} \mathrm{P}$ labeled RNA probe derived from $\beta$ cardiac MHC gene sequences (6). RNase A digests hybrid molecules containing mismatched base pairs. Unaffected individuals $(P)$ contain the 171-bp fragment, which is cleaved into 130- and 41-bp fragments in affected individuals. 
tions (Arg719Trp and Gly716Arg) change the charge of the encoded amino acid, while one (Phe513Cys) is a conservative mutation. Affected individuals from four families bearing the Arg719Trp mutation have a marked reduction in life expectancy, while affected individuals with the Phe513Cys mutation have a near normal survival. These analyses add to the growing body of information regarding the effect that a particular $\beta$ cardiac MHC gene mutation has on clinical outcome in FHC.

$15 \beta$ cardiac MHC missense mutations that cause FHC have been identified (Fig. 5). A wide range in the distribution and severity of hypertrophy has been observed in affected individuals with the same mutation (3, 5, Table I, and our unpublished data ) suggesting a multifactorial etiology to the morphologic features of this disorder. In contrast, analyses of the natural history of FHC in large kindreds and in unrelated families with a common mutation demonstrate that genotype can substantially influence survival. We suggest that $\beta$ cardiac MHC missense mutations can be divided into three groups (Fig. 5): benign $(B)$ or those associated with near normal life expectancy, malignant $(M)$ or those which significantly reduce life expectancy, and intermediate $(I)$ or those with a moderate effect on life expectancy.

Three benign mutations have been identified, located in the head region (Phe513Cys, exon 15; Val606Met, exon 16 [6]) or in the head/rod junction (Leu908Val, exon 23 [7, 16]). Each of these is a conservative mutation, suggesting that the lack of charge change may in part account for the good prognosis associated with these mutations. Regional localization may also contribute to a benign phenotype. None of the mutations characterized as malignant occur in the head/rod junction and the sole mutation identified in the rod (a $3^{\prime}$ deletion [17]) produces a strikingly late onset of disease. Collectively, these data suggest that mutations which arise within the head/rod or rod region are more likely to be benign or may be clinically silent.

Significant premature mortality accompanies three mutations: Arg403Gln (exon 13 [15]), Arg453Cys (exon 14 [6]), or Arg719Trp (exon 19). In contradistinction to that observed in myosin mutations of the nematode (18), there appears to be little clustering of malignant mutations within the head region of the $\beta$ cardiac MHC gene. While none of the malignant FHC mutations directly alter sequences that appear critical for binding ATP or myosin light chains, the recently published crystal structure of the chicken skeletal muscle myosin head by Rayment and colleagues (23) provides insight into the consequences of one malignant mutation. The three-dimensional structure revealed a loop formed by amino acid residue Arg 405 (corresponding to amino acid residue 403 in human) to residue Lys 415, that interacts with actin. The Arg403Gln mutation should alter loop structure and effect stereospecific interactions between actin and myosin. The three-dimensional structure of myosin combined with biochemical studies (24, 25 ) suggested a model for muscle contraction in which conformational changes in myosin occur during ATP binding and hydrolysis that disrupts actin binding. Actin and myosin rebinding is hypothesized to be sequential and requires several conformational states for myosin that produce stereospecific interactions between these molecules. This model suggests that the location of a mutation and resultant changes in stoichiometry and charge may account for differing muscle function that ultimately impact on survival. We speculate that malignant mutations either impede conformational changes in myosin or alter interactions with actin and other molecules. Less severe phenotypes are expected from mutations that are either positioned in noncritical regions or that produce modest changes in
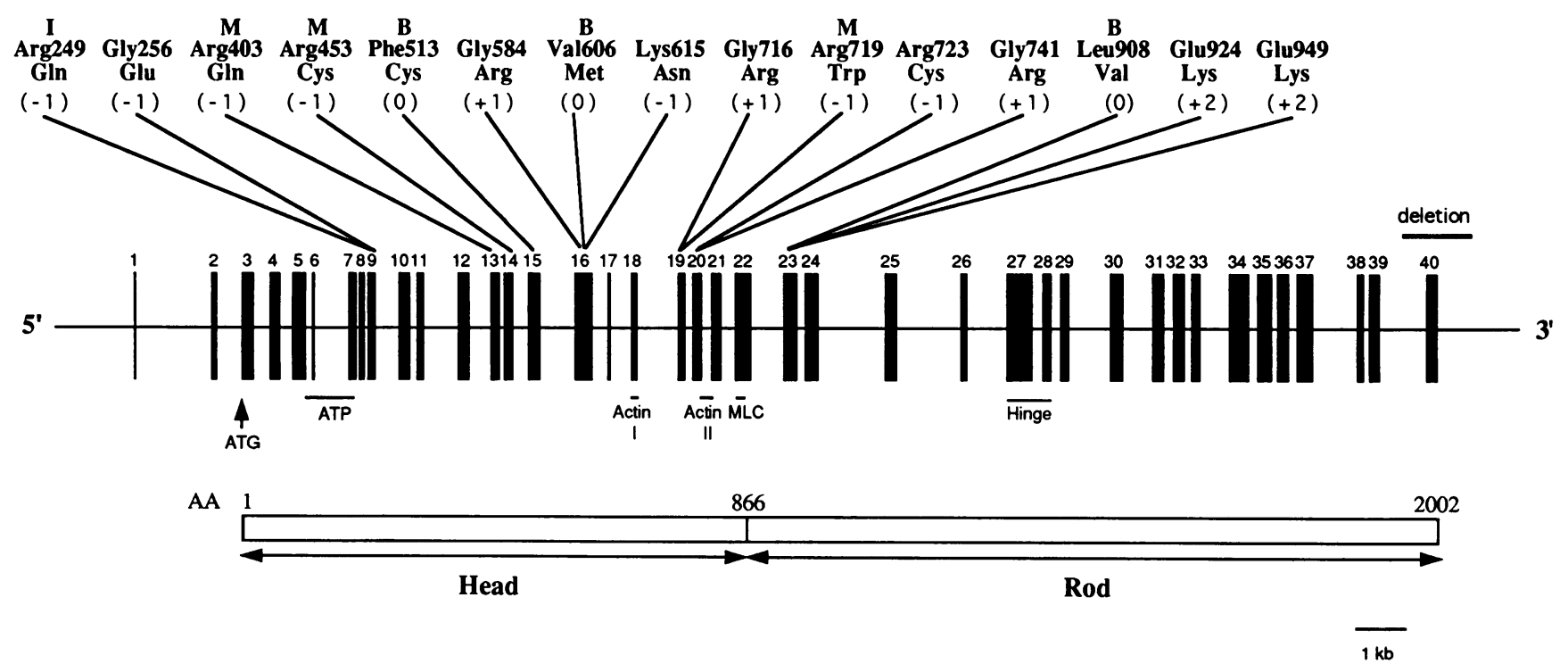

Figure 5. A schematic of mutations within the $\beta$ cardiac MHC gene that cause FHC. Mutations that are previously described (Arg249Gln, 9; Gly256Glu, 19; Arg403Gln, 6, 7, 15, 18, 20; Arg453Cys, 6; Gly584Arg, 6; Val606Met, 6; Lys615Asn, 21; Arg723Cys, 22; Gly741 Arg, 19; Leu908Val, 7, 16; Glu924Lys, 6; Glu949Lys, 6; deletion, 16) and reported here (Phe513Cys, Gly716Arg, Arg719Trp) are shown by a three-letter code to denote the normal and mutated amino acid sequence. The change in charge resulting from each mutation is indicated. The letter $B$ (benign) denotes mutations associated with near normal life expectancy; $\mathbf{M}$ (malignant) denotes those which significantly reduce life expectancy; I (intermediate) indicates mutations with a moderate effect on life expectancy. The transcription initiation signal (ATG) and regions that encode the binding of ATP, actin, or the myosin light chain (MLC) are indicated. The globular head and rod segment of the $\beta$ cardiac MHC polypeptide are shown. 
changes in secondary structure. Alternatively, some mutations may perturb a single myosin function, whereas other mutations may alter several functions and result in a more severe phenotype. Future studies that integrate genetic data, structural modeling, and biochemical studies should more fully elucidate the mechanisms by which mutations perturb myosin physiology and result in significant cardiac pathology.

Definition of the full spectrum of $\beta$ cardiac MHC mutations that cause the differing phenotypes found in FHC will contribute to understanding myosin processes. Ultimately this may help to improve therapeutics for the dysfunctional hypertrophic heart. More immediately, a compilation of the clinical outcomes associated with different $\beta$ cardiac MHC mutations will enable physicians to recognize and treat those individuals at risk for the serious sequelae of FHC.

\section{Acknowledgments}

This study would not have been possible without the invaluable assistance of family members. We are grateful to Rita Hill and Annie O'Donoghue for assembling data on family members, and to Mohammad Miri for technical assistance.

This work was supported by grants from the Howard Hughes Medical Foundation (to R. Anan and J. G. Seidman), Deutsche Forschungsgemeinschaft (to L. Thierfelder), British Heart Foundation (to H. Watkins and W. J. McKenna), the Ministry of Health and Welfare of Japan (to H. Tanaka), the US National Institutes of Health (P50HL-42267 to R. Roberts; HL-46320 to J. G. Seidman; HL-42467 to C. E. Seidman), the American Heart Association (International Research Fellow, G. Greve; Established Investigator, C. E. Seidman), and the Bristol Myers Squibb Company (to J. G. Seidman and C. E. Seidman).

\section{References}

1. Watkins, H., C. MacRae, L. Thierfelder, Y.-H. Chou, M. Frenneaux, W. McKenna, J. G. Seidman, and C. E. Seidman. 1993. A disease locus for familial hypertrophic cardiomyopathy maps to chromosome Iq3. Nature Genet. 3:333337.

2. Carrier, L., C. Hengstenberg, J. S. Beckmann, P. Guicheney, C. Dufour, J. Bercovici, E. Dausse, I. Berebbi-Bertrand, C. Wisnewsky, D. Pulvenis, L. Fetler, A. Vignal, J. Weissenbach, D. Hillaire, J. Feingold, J.-B. Bouhour, A. Hagege, M. Desnos, R. Isnard, O. Duibourg, M. Komajda, and K. Schwartz. 1993. Mapping a novel gene for familial hypertrophic cardiomyopathy to chromosome 11 . $\mathrm{Na}$ ture Genet. 4:311-313.

3. Jarcho, J. A., W. McKenna, P. Pare, S. D. Solomon, R. F. Holcombe, S. Dickie, T. Levi, H. Donis-Keller, J. G. Seidman, and C. E. Seidman. 1989. Mapping a gene for familial hypertrophic cardiomyopathy to chromosome $14 \mathrm{ql}$. $N$ Engl. J. Med. 321:1372-1378.

4. Thierfelder, L., C. MacRae, H. Watkins, J. Tomfohrde, W. McKenna, K. Bohm, G. Noeske, M. Schlepper, J. Just, A. Bowcock, H.-P. Vosberg, C. Seidman, and J. G. Seidman. 1993. A disease locus for familial hypertrophic cardiomyopathy maps to chromosome 15q2. Proc. Natl. Acad. Sci. USA. 90:62706274.

5. Solomon S. D., S. Wolff, H. Watkins, P. M. Ridker, P. C. Come, C. E. Seidman, W. J. McKenna, and R. T. Lee. 1993. Left ventricular hypertrophy and morphology in familial hypertrophic cardiomyopathy associated with mutations of the $\beta$-myosin heavy chain gene. J. Am. Coll. Cardiol. 22:498-505.

6. Watkins, H., A. Rosenzweig, D.-S. Hwang, T. Levi, W. McKenna, C. E. Seidman, and J. G. Seidman. 1992. Characteristics and prognostic implications of myosin missense mutations in familial hypertrophic cardiomyopathy. $N$. Engl. J. Med. 326:1108-1114.

7. Epstein, N. D., G. M. Cohn, F. Cyran, and L. Famnanapazir. 1992. Differences in clinical expression of hypertrophic cardiomyopathy associated with two distinct mutations in the $\beta$-myosin heavy chain gene; a 908Leu $\rightarrow$ Val mutation and a $403 \mathrm{Arg} \rightarrow$ Gin mutation. Circulation. 86:345-352.

8. Hejtmancik, J. F., P. A. Brink, J. Towbin, R. Hill, L. Brink, T. Tapscott, A. Trakhtenbriot, and R. Roberts. 1991. Localization of gene for familial hypertrophic cardiomyopathy to chromozome $14 \mathrm{q} 1$ in a diverse US population. Circulation. 83:1592-1597.

9. Rosenzweig, A., H. Watkins, D.-S. Hwang, M. Miri, W. McKenna, T. A. Trail, J. G. Seidman, and C. E. Seidman. 1991. Preclinical diagnosis of familial hypertrophic cardiomyopathy by genetic analysis of blood lymphocytes. $N$. Engl. J. Med. 325:1753-1760.

10. Kogan, S. C., M. Doherty, and J. Gitschier. 1987. An improved method for prenatal diagnosis of genetic diseases by analysis of amplified DNA sequences. Application to hemophilia A. N. Engl. J. Med. 317:985-990.

11. Cotton, R. G. H., N. R. Rodrigues, and R. D. Campbell. 1988. Reactivity of cytosine and thymine in single-base-pair mismatches with hydroxylamine and osmium tetroxide and its application to the study of mutations. Proc. Natl. Acad. Sci. USA. 85:4397-4401.

12. Jaenicke, T., K. W. Diederich, W. Haas, J. Schleich, P. Lichter, and H.-P. Vosberg. 1990. The complete sequence of the human $\beta$-myosin heavy chain gene and an analysis of its product. Genomics. 8:194-206.

13. Treger, A., and S. G. Blout. 1965. Familial cardiomyopathy. Am. Heart J. 70:40-53.

14. Paul, G., T. J. Richer, W. J. Gallen, and D. Z. Friedberg. 1972. Familial cardiomyopathy in a Milwaukee family. Wisc. Med. J. 71:198-202.

15. Geisterfer-Lowlence, A. A. T., S. Kass, G. Tanigawa, H.-P. Vosberg, W. McKenna, C. E. Seidman, and J. G. Seidman. 1990. A molecular basis for familial hypertrophic cardiomyopathy: A $\beta$ cardiac myosin heavy chain gene missense mutation. Cell. 62:999-1006.

16. Al-Mahdawi, S., S. Chamberlain, J. Cleland, P. Nihoyannopoulos, D. Gilligan, J. French, L. Choudhury, R. Williamson, and C. Oakley. 1993. Identification of a mutation in the $\beta$ cardiac myosin heavy chain gene in a family with hypertrophic cardiomyopathy. Br. Heart J. 69:136-141.

17. Marian, A. J., Q.-T. Yu, A. Mares, Jr., R. Hill, R. Roberts, and M. B. Perryman. 1992. Detection of a new mutation in the $\beta$-myosin heavy chain gene in an individual with hypertrophic cardiomyopathy. J. Clin. Invest. 90:21562165 .

18. Bejsovec, A., and P. Anderson. 1990. Functions of the myosin ATP and actin binding sites are required for $\mathrm{C}$. elegans thick filament assembly. Cell. 60:133-140.

19. Fananapazir, L., M. C. Dalakas, C. Francis, C. Gabriel, and N. D. Epstein. 1993. Missense mutations in the $\beta$-cardiac myosin heavy-chain gene cause central core disease in hypertrophic cardiomyopathy. Proc. Natl. Acad. Sci. USA. 90:3993-3997.

20. Perryman, M. B., Q.-T. Yu, A. J. Marian, A. Mares, Jr., G. Czernuszewicz, J. Ifegwu, R. Hill, and R. Roberts. 1992. Expression of a missence mutation in the messenger RNA for $\beta$-myosin heavy chain in myocardial tissue in hypertrophic cardiomyopathy. J. Clin. Invest. 90:271-277.

21. Nishi, H., A. Kimura, H. Harada, H. Toshima, and T. Sasazuki. 1992. Novel missence mutation in cardiac $\beta$ myosin heavy chain gene found in a Japanese patient with hypertrophic cardiomyopathy. Biochem. Biophys. Res. Commun. 188:379-387.

22. Watkins, H., L. Thierfelder, D.-S. Hwang, W. McKenna, J. G. Seidman, and C. E. Seidman. 1992. Sporadic hypertrophic cardiomyopathy due to de novo myosin mutations. J. Clin. Invest. 90:1666-1671.

23. Rayment, I., W. R. Rypniewski, K. Schmidt-Base, R. Smith, D. R. Tomchick, M. M. Benning, D. A. Winkelmann, G. Wesenberg, and H. M. Holden. 1993. Three-dimensional structure of myosin subfragment-1: a molecular motor. Science (Wash. DC). 261:50-58.

24. Rayment, I., H. M. Holden, M. Whittaker, C. B. Yohn, M. Lorenz, K. C. Holmes, and R. A. Millian. 1993. Structure of the actin-myosin complex and its implications for muscle contraction. Science (Wash. DC). 261:58-65.

25. Cuda, G., L. Fananapazir, W.-S. Zhu, J. R. Sellers, and N. D. Epstein. 1993. Skeletal muscle expression and abnormal function of $\beta$-myosin in hypertrophic cardiomyopathy. J. Clin. Invest. 91:2861-2865. 